\title{
Adiabatic dynamics in passage across quantum critical lines and gapless phases
}

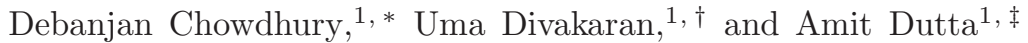 \\ ${ }^{1}$ Department of Physics, Indian Institute of Technology, Kanpur 208 016, India
}

(Dated: October 30, 2018)

\begin{abstract}
It is well known that the dynamics of a quantum system is always non-adiabatic in passage through a quantum critical point and the defect density in the final state following a quench shows a power-law scaling with the rate of quenching. However, we propose here a possible situation where the dynamics of a quantum system in passage across quantum critical regions is adiabatic and the defect density decays exponentially. This is achieved by incorporating additional interactions which lead to quantum critical behavior and gapless phases but do not participate in the time evolution of the system. To illustrate the general argument, we study the defect generation in the quantum critical dynamics of a spin-1/2 anisotropic quantum XY spin chain with three spin interactions and a linearly driven staggered magnetic field.

PACS numbers: 05.30.-d,64.60.-i,75.10.-b
\end{abstract}

Understanding the dynamics of a quantum system passing through a quantum critical point [1, 2] has been a very active and fascinating area of research in recent years [3 14]. The dynamical evolution can be initiated either by a sudden change of a parameter in the Hamiltonian, called a sudden quench [3], or by a slow (adiabatic) quenching of a parameter [4,5]. The related entanglement and fidelity properties are also being looked at [15]. It is well known that when a quantum system, initially prepared in its ground state, is swept adiabatically across a quantum critical point, defects are generated in the final state of the system due to the critical slowing down which forces the dynamics of the system to be non-adiabatic in the vicinity of the quantum critical point. In a linear passage through an isolated critical point, when a parameter (e.g., the magnetic field) is changed in time $t$ as $t / \tau$, the defect density $(n)$ in the final state scales with the quenching rate $1 / \tau$ following the Kibble-Zurek scaling relation [16, 17] given by $n \sim \tau^{-d \nu /(\nu z+1)}$ in the adiabatic limit $(\tau \rightarrow \infty)[4$, [5, 12]. Here, $d$ is the spatial dimension, $\nu$ and $z$ are the correlation length and the dynamical exponents, respectively, associated with the quantum critical point [1]. The experimental verification of such dynamics is now possible by studying the dynamics of atoms trapped in optical lattices [18].

In this work, we explore the possibility of adiabatic dynamics or exponentially decaying defect density even in passage through quantum critical points and gapless phases. We show that this occurs only in a special situation in which an additional term of the Hamiltonian that leads to the quantum critical behavior and gapless phases does not participate in the dynamics of the system. We illustrate the general argument using a quantum spin- $1 / 2$ $\mathrm{XY}$ chain with multispin interactions and a staggered magnetic field which is exactly solvable via the Jordan-

\footnotetext{
*Electronic address: debanjanchowdhury@gmail.com

$\dagger$ Electronic address: udiva@iitk.ac.in

${ }^{\ddagger}$ Electronic address: dutta@iitk.ac.in
}

Wigner transformation. We thus provide an example of a special situation that contradicts the power-law scaling of the defect density for a non-random quantum system [4, 5].

Let us begin the discussion with a $d$-dimensional quantum Hamiltonian given by

$$
\begin{aligned}
H & =\sum_{k} \psi^{\dagger}(\vec{k}) H(\vec{k}) \psi(\vec{k}) ; \\
H(\vec{k}) & =\alpha c(\vec{k}) \hat{1}+\left(\lambda \sigma^{z}+\Delta(\vec{k}) \sigma_{+}+\Delta^{*}(\vec{k}) \sigma_{-}\right)
\end{aligned}
$$

where $\alpha, c(\vec{k})$ and $\Delta(\vec{k})$ are model dependent functions, $\sigma^{i}(i=x, y, z)$ are Pauli spin matrices, $\sigma_{+}=\left(\sigma^{x}+i \sigma^{y}\right) / 2$, $\sigma_{-}=\left(\sigma^{x}-i \sigma^{y}\right) / 2$ and $\hat{1}$ denotes the $2 \times 2$ identity matrix. Here, $\lambda$ defines the time-dependent parameter that is to be quenched adiabatically. The column vector $\psi(\vec{k})$ defines a two component fermionic operator. Such an exactly solvable Hamiltonian (with $\alpha=0$ ) is known to represent several one- and two-dimensional integrable quantum spin models as the Ising, the XY spin chains 19] and the extended Kitaev model in two dimensions 20] when the spin operators are transformed into spinless fermions via the Jordan-Wigner transformation [19]. In the present case, however, the nature of interaction of the spin chains we study, necessitates the consideration of a two sublattice structure, and hence the fermion operator $\psi(\vec{k})=\left(a_{\vec{k}}, b_{\vec{k}}\right)$ where $a_{\vec{k}}\left(b_{\vec{k}}\right)$ denote the Jordan-Wigner Fermions for the mode $\vec{k}$ describing the spins on even (odd) sublattices. The excitation energy of the Hamiltonian is given by

$\epsilon_{\vec{k}}^{ \pm}=\alpha c(\vec{k}) \pm \sqrt{\lambda^{2}+|\Delta|^{2}}$.

The phase diagram of the model in the $\alpha-\lambda$ plane can be easily obtained. The presence of the additional term $\alpha c(\vec{k})$ plays a non-trivial role in determining the phase diagram of the model by making excitation energy zero for specific values of the parameter and the wavevector $\vec{k}$. For example, if $\Delta(\vec{k})=0$ for the wavevector $\vec{k}_{0}$, we obtain a critical line $\left(\epsilon_{\vec{k}}^{-}=0\right)$ given by $\lambda=\alpha c\left(\vec{k}_{0}\right)$. 
Our interest is in the defect generation when the parameter $\lambda$ is quenched in a linear fashion as $t / \tau$ from $t \rightarrow-\infty$ to $t \rightarrow+\infty$ and the system is swept across the critical line. Let us assume that at $t \rightarrow-\infty$, the system is prepared in its ground state $\left|1_{\vec{k}}\right\rangle$ such that $a_{\vec{k}}^{\dagger} a_{\vec{k}}\left|1_{\vec{k}}\right\rangle=1$ and $b_{\vec{k}}^{\dagger} b_{\vec{k}}\left|1_{\vec{k}}\right\rangle=0$ for any $\vec{k}$. For an adiabatic dynamics, the expected final state is $\left|2_{\vec{k}}\right\rangle$ defined as $b_{\vec{k}}^{\dagger} b_{\vec{k}}\left|2_{\vec{k}}\right\rangle=1$. In course of dynamics, a general state describing the system at an instant $t$ can be put in the form $\psi_{\vec{k}}(t)=C_{1, \vec{k}}(t)\left|1_{\vec{k}}\right\rangle+C_{2, \vec{k}}(t)\left|2_{\vec{k}}\right\rangle$ where the time dependent coefficients satisfy the Schrodinger equation

$$
\begin{aligned}
i \frac{\partial}{\partial t}\left(\begin{array}{c}
C_{1, \vec{k}} \\
C_{2, \vec{k}}
\end{array}\right) & =H_{\vec{k}}(t)\left(\begin{array}{c}
C_{1, \vec{k}} \\
C_{2, \vec{k}}
\end{array}\right) \\
& =\left(\begin{array}{cc}
\lambda(t) & \Delta(\vec{k}) \\
\Delta(\vec{k})^{*} & -\lambda(t)
\end{array}\right)\left(\begin{array}{l}
C_{1, \vec{k}} \\
C_{2, \vec{k}}
\end{array}\right)
\end{aligned}
$$

with $\hbar$ set equal to unity. Also, the time dependent parameter $\lambda(t)$ varies as $\lambda_{0} t / \tau$ and the initial conditions are $\left|C_{1, \vec{k}}(t \rightarrow-\infty)\right|^{2}=1$ and $\left|C_{2, \vec{k}}(t \rightarrow-\infty)\right|^{2}=0$. It is worth noting that although the term $\alpha c(\vec{k}) \hat{1}$ plays a crucial role in determining the critical line or the gapless regions, it does not show up in the time-dependent Hamiltonian $H_{\vec{k}}(t)$ which dictates the temporal evolution. This is because the identity operator commutes with all the other terms of the Hamiltonian at every instant of time. The term $\alpha c(\vec{k}) \hat{1}$ influences the dynamics only up to a phase factor and is hence truly irrelevant in deciding the non-adiabatic behavior. In this sense, the time dependent Hamiltonian $H_{\vec{k}}(t)$ does not capture the passage through quantum critical lines and gapless phases generated by $\alpha c(\vec{k})$ ! The Schrodinger equations (3) describing the dynamics of the system effectively boils down to the standard Landau-Zener problem (LZ) 21] of two timedependent levels $\pm \sqrt{\lambda(t)^{2}+|\Delta|^{2}}$ (not the levels given in Eq. 2) approaching each other in a linear fashion with a minimum gap $2|\Delta|$ at time $t=0$. The probability of excitation in the final state is given by Landau Zener formula [21, 22] $p_{\vec{k}}=\left|C_{1, \vec{k}}(t \rightarrow \infty)\right|^{2}=\exp \left(-\left(\pi \Delta(\vec{k})^{2} \tau\right) / \lambda_{0}\right)$.

Let us assume that the parameter $\Delta(\vec{k})$ vanishes at a quantum critical point for $\alpha=0$ as $\Delta(\vec{k}) \sim\left|\vec{k}-\vec{k}_{0}\right|^{z_{2}}$ where $\vec{k}_{0}$ is the critical wave vector. Noting that in the adiabatic limit of large $\tau$, only modes close to $\vec{k}_{0}$ contribute, the defect density in the final state is given by

$$
\begin{aligned}
n & =\frac{1}{(2 \pi)^{d}} \int_{B Z} p_{k} d^{d} k \\
& =\frac{1}{(2 \pi)^{d}} \int_{B Z} d^{d} k \exp \left(-\left(\pi\left|\vec{k}-\vec{k}_{0}\right|^{2 z_{2}} \tau\right) / \lambda_{0}\right) \\
& \sim \frac{1}{\tau^{d / 2 z_{2}}}
\end{aligned}
$$

The scaling of the density of defects hence depends only on the exponent $z_{2}$ as observed previously in references [11] in the context of quenching through a multicritical point. The situation where the parameter $\Delta(\vec{k})$ does never vanish during the temporal evolution of $H_{\vec{k}}(t)$ is far more interesting. If the parameter $\Delta$ attains the minimum value $\Delta_{0}$ for some wave vector $k_{0}$ satisfying the scaling form $\Delta^{2}=\Delta_{0}^{2}+\delta\left|\vec{k}-\vec{k}_{0}\right|^{2 z_{2}}$, the nonadiabatic transition probability will show an exponential behavior and the defect density will scale as $n \sim$ $\exp \left(-\pi \Delta_{0}^{2} \tau / \lambda_{0}\right) / \tau^{d / 2 z_{2}}$. As discussed already, the scaling of the defect density satisfy the same scaling form for all values of $\alpha$. This exponential decay of defect even in passage through a quantum critical point is the key feature of this communication.

Question is whether it is possible to find a Hamiltonian which gets mapped to the Eq. (1). To show this we consider a spin-1/2 quantum XY spin with a two sublattice structure in the presence of a three-spin interaction and a staggered magnetic field $h$ given by the Hamiltonian

$$
\begin{aligned}
& H=-h \sum_{i}\left(\sigma_{i, 1}^{z}-\sigma_{i, 2}^{z}\right)-J_{1} \sum_{i}\left(\sigma_{i, 1}^{x} \sigma_{i, 2}^{x}+\sigma_{i, 1}^{y} \sigma_{i, 2}^{y}\right) \\
& -J_{2} \sum_{i}\left(\sigma_{i, 2}^{x} \sigma_{i+1,1}^{x}+\sigma_{i, 2}^{y} \sigma_{i+1,1}^{y}\right)-J_{3} \sum_{i}\left(\sigma_{i, 1}^{x} \sigma_{i, 2}^{z} \sigma_{i+1,1}^{x}\right. \\
& \left.+\sigma_{i, 1}^{y} \sigma_{i, 2}^{z} \sigma_{i+1,1}^{y}\right)-J_{3} \sum_{i}\left(\sigma_{i, 2}^{x} \sigma_{i+1,1}^{z} \sigma_{i+1,2}^{x}\right. \\
& \left.+\sigma_{i, 2}^{y} \sigma_{i+1,1}^{z} \sigma_{i+1,2}^{y}\right),
\end{aligned}
$$

where $i$ is the site index and the additional subscript 1(2) defines the odd (even) sublattice. The parameter $J_{1}$ describes the XY interaction between spins on sublattice 1 and 2 while $J_{2}$ describes the XY interaction between spins on sublattice 2 and 1 such that $J_{1}$ is not necessarily equal to $J_{2}$. The parameter $J_{3}$, chosen to be positive throughout, denotes the three spin interaction. Some variants of the Hamiltonian (5) were studied previously [23]. This spin chain is exactly solvable in terms of Jordan-Wigner fermions [23] defined on even and odd sublattices as $\sigma_{i, 1}^{+}=\left[\prod_{j<i}\left(-\sigma_{j, 1}^{z}\right)\left(-\sigma_{j, 2}^{z}\right)\right] a_{i}^{\dagger}$, and $\sigma_{i, 2}^{+}=$ $\left[\prod_{j<i}\left(-\sigma_{j, 1}^{z}\right)\left(-\sigma_{j, 2}^{z}\right)\left(-\sigma_{i, 1}^{z}\right)\right] b_{i}^{\dagger}$, where $\sigma_{i, 1}^{z}=2 a_{i}^{\dagger} a_{i}-1$ and $\sigma_{i, 2}^{z}=2 b_{i}^{\dagger} b_{i}-1$. The Fermion operators $a_{i}$ and $b_{i}$ can be shown to satisfy Fermionic anticommutation relations.

In terms of the Jordan-Wigner Fermions, the Hamiltonian (5) can be recast in the Fourier space to the form given in Hamiltonian (1) with

$$
H_{k}=\alpha \cos k \hat{1}-\frac{1}{2}\left[\begin{array}{cc}
\lambda & -\left(1+\gamma e^{-i k}\right) \\
-\left(1+\gamma e^{+i k}\right) & -\lambda
\end{array}\right],
$$

where $\psi_{k}^{\dagger}=\left(a_{k}^{\dagger}, b_{k}^{\dagger}\right)$ and we have set $\lambda=h / J_{1}, \alpha=J_{3} / J_{1}$ and $\gamma=J_{2} / J_{1}$. The excitation energy is obtained as

$\epsilon_{k}^{ \pm}=\alpha \cos k \pm \frac{1}{2} \sqrt{\lambda^{2}+1+\gamma^{2}+2 \gamma \cos k}$

Comparing with the Hamiltonian (1), we also identify $c(k)=\cos k$ and $|\Delta(k)|^{2}=1+\gamma^{2}+2 \gamma \cos k$. The phase 

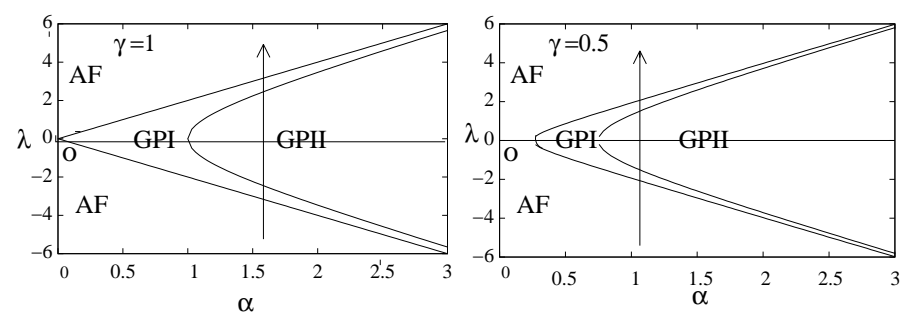

FIG. 1: Phase diagram of the Hamiltonian (5) in the $\alpha-\lambda$ plane for $\gamma=1$ and $\gamma=0.5$. Different phases are discussed in the main text. The vertical line shows the direction of quenching.

diagram of the model for both $\gamma=1$ and $\gamma \neq 1$ are shown in Fig. 1. For $\gamma \neq 1$, excitation energy $\epsilon_{k}^{+}$vanishes for the mode $k=\pi$ at the phase boundary between an antiferromagnetic phase (AF) and a gapless phase (GPI) given by $2 \alpha=\sqrt{\lambda^{2}+(1-\gamma)^{2}}$. In the GPI phase, it is always possible to find a wave vector $k$ for which $\epsilon_{k}^{+}$ vanishes. Similarly, $\epsilon_{k}^{-}$vanishes for the mode $k=0$ at the phase boundary given by $2 \alpha=\sqrt{\lambda^{2}+(1+\gamma)^{2}}$ which marks the boundary between the GPI and the second gapless phase (GPII). In GPII, both $\epsilon_{k}^{+}$and $\epsilon_{k}^{-}$vanish for some wave vector, so that there are four Fermi points in contrast to two Fermi points in GPI. The transition between GPI and GPII phases is a special quantum phase transition that involves doubling the number of Fermi points 24]. For $\gamma=1$, on the other hand, we arrive at a simplified form $|\Delta(k)|^{2}=2+2 \cos k=4 \cos ^{2}(k / 2)$. The phase boundaries between the antiferromagnetic phase and GPI phase, and the GPI and GPII phase are given by $2 \alpha=\lambda$ and $2 \alpha=\sqrt{\lambda^{2}+4}$, respectively. It is noteworthy that for the case $\gamma=1$, the parameter $|\Delta|^{2}$ vanishes at the AF-GPI phase boundary for $k=\pi$ and any $\alpha$ whereas in the anisotropic case, never does it vanish at the quantum transitions!

In the quenching scheme we employ here, the scaled staggered magnetic field $\lambda$ is quenched as $\lambda_{0} t / \tau$ from $t \rightarrow$ $-\infty$ to $+\infty$ with $\alpha \neq 0$ so that the system is swept across the quantum critical lines and the gapless phases. Let us set $\lambda_{0}=1$ for simplicity. As discussed already, so far as the dynamics is concerned, the term $\alpha \cos k$ is irrelevant and the Schrodinger equation essentially reduces to a two level LZ problem. Note that in both the limits $\lambda \rightarrow$ $\pm \infty$, the spins should be in a perfect antiferromagnetic orientation in the $z$-direction and wrongly oriented spins in the final state at $t \rightarrow \infty(\lambda \rightarrow \infty)$ are the defects.

Using the LZ transition formula we find that the probability of excitation in the final state is given by $p_{k}=\exp \left(-\pi|\Delta(k)|^{2} \tau\right)=\exp \left(-4 \pi \cos ^{2}(k / 2) \tau\right)$ for $\gamma=1$. In the adiabatic limit of $\tau \rightarrow \infty$, only the modes close to $k=\pi$ (for which $|\Delta(k)|^{2}$ vanishes) contribute, so that $p_{k}$ takes the simplified from $p_{k}=\exp \left(-\pi(\pi-k)^{2} \tau\right)$, and
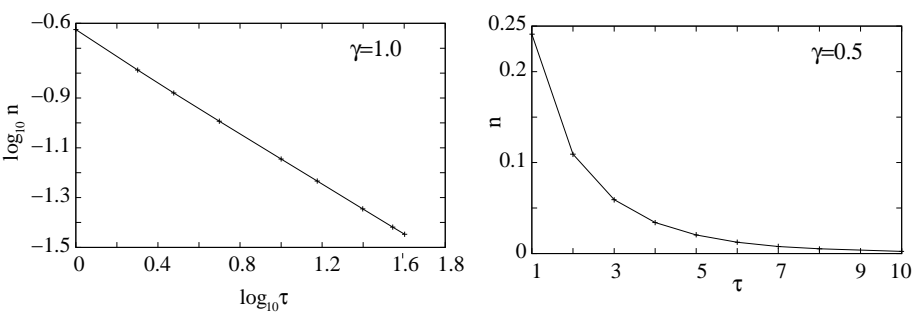

FIG. 2: Numerical integration result for the defect density as a function of $\tau$ for $\gamma=1$ and $\gamma=0.5$. The parameter $\alpha$ is chosen such that the system crosses both the gapless phases in the two cases. For $\gamma=1$, we have used log-scale and slope of the straight line is $1 / 2$ indicating a power-law decay of the defect density. For $\gamma=0.5$, we have used linear scale to accentuate the exponential fall of the defect density.

hence the defect density in the final state is given by

$n=\frac{1}{\pi} \int_{0}^{\pi} d k \exp \left(-\pi(\pi-k)^{2} \tau\right) \sim \frac{1}{\sqrt{\tau}}$.

For $\gamma \neq 1$, on the other hand, $\Delta$ is minimum for $k=\pi$ on the AF-GPI phase boundary given by $\left|\Delta_{0}\right|^{2}=|1-\gamma|^{2}$ so that the defect density

$$
\begin{aligned}
n & =\frac{1}{\pi} \int_{0}^{\pi} d k \exp \left(-\pi\left|1+\gamma^{2}+2 \gamma \cos k\right| \tau\right) \\
& \sim \frac{e^{-\pi\left|\Delta_{0}\right|^{2} \tau}}{\sqrt{\tau}} .
\end{aligned}
$$

We therefore, come across a special situation where the defect density decays exponentially with the rate $\tau$ even though the system is swept across the critical lines and gapless phases as long as $\Delta_{0} \neq 0$, i.e., $\gamma \neq 1$. We recover the power law scaling in $\mathrm{Eq}(8)$ for $\gamma=1$. As mentioned already, the scaling behavior shown in Eqs. (8) and (9) are same for all values of the scaled three spin interaction $\alpha$. The defect density obtained by numerical integration of the Schrodinger equations (3) is shown in Fig 2.

It is to be noted that the time evolution governed by $H_{k}(t)$ is completely insensitive to the phase transitions generated by $\alpha c(k)$. This fact can be understood also using the following argument: the total number of Fermions for a mode $k$ is a constant of motion as far as the LandauZener dynamics is concerned. This is because the number operator $n_{k}=a_{k}^{\dagger} a_{k}+b_{k}^{\dagger} b_{k}$ commutes with $H_{k}(t)$ for all $k$. In the initial (final) state $\lambda \rightarrow-\infty(+\infty)$, the expectation value of $n_{k}$ is unity which as per the above argument is conserved throughout the dynamics. On the other hand, the three spin term gives rise to the gapless phases where both the energies $\epsilon_{k}^{+}$and $\epsilon_{k}^{-}$can become negative or both can become positive for some values of $k$; the true ground state allows $n_{k}=2$ or 0 in those cases. However, the instantaneous eigenstates of the time-dependent Hamiltonian will continue to have $n_{k}=1$ as explained above and does not reach the true ground state. 
Question remains whether the result presented here should persist in the case of a general interacting system. In ref. [5], it was shown that the proof of the Kibble-Zurek scaling form does not require the system to be broken up into a product of two-level systems which can then be analyzed by Landau-Zener tunneling formula. The argument only uses translational invariance and some general scaling arguments, namely, the momentum dependence of the energies and the parameter dependence of the wave functions of those states. Extending the argument of ref. [5] in the present case demands that the Hamiltonian should be decoupled into two parts; one time independent $H_{1}(\alpha)$ and the other time dependent $H_{2}(\lambda(t))$, where $\lambda=t / \tau$ as defined before, and $H_{1}$ commutes with all the terms of $H_{2}(\lambda)$. It can be shown that $H_{1}(\alpha)$ does not influence the dynamics except for a phase factor and the scaling form of the density of defects is then given by scaling form of the $\lambda$-dependent part of the excitation energy $\delta \omega_{2, k}(\lambda)$ whereas vanishing of the total excitation energy $\delta \omega_{k}=\delta \omega_{1, k}(\alpha)+\delta \omega_{2, k}(\lambda)$ leads to the complex phase diagram with critical lines and gapless phases. If $\delta \omega_{2, k}(\lambda)$ does not vanish, one expects an exponential de- cay of the defect density. On the other hand, if $\delta \omega_{2, k}$ vanishes for any mode $k$, then the density of defects decays as a powerlaw with the quenching rate $\tau$. In the present work, we have used an example of an exactly solved system which satisfies the above conditions. Though possible in principle, we believe that finding the example of such an interacting Hamiltonian which satisfies the above conditions and show an exponential decay of the defect density is a difficult and open problem. Our interest in this work is only to point out a special situation where one can find exponential decay of defect density even during passage through critical regions and we have illustrated the possibility using an exactly solvable spin chain with complicated interactions.

Acknowledgement We acknowledge Diptiman Sen and G.E. Santoro for very interesting and critical comments and Victor Mukherjee for carefully reading the manuscript. D.C. acknowledges KVPY, Department of Science and Technology (Government of India) for support and also G. Refael for interesting discussions. AD and UD acknowledge Abdus Salam ICTP, Trieste, Italy, where some part of this work was done.
[1] S. Sachdev, Quantum Phase Transitions (Cambridge University Press, Cambridge, 1999).

[2] B. K. Chakrabarti, A. Dutta, and P. Sen, Quantum Ising Phases and Transitions in Transverse Ising Models, m41 (Springer-Verlag, Berlin, 1996).

[3] T. Kadowaki and H. Nishimori, Phys. Rev. E 58, 5355 (1998); K. Sengupta, S. Powell, and S. Sachdev, Phys. Rev. A 69, 053616 (2004); A. Das, K. Sengupta, D. Sen, and B. K. Chakrabarti, Phys. Rev. B 74, 144423 (2006); P. Calabrese and J. Cardy, J. Stat. Mech: Theory Expt P04010 (2005), and Phys. Rev. Lett. 96, 136801 (2006);

[4] W. H. Zurek, U. Dorner, and P. Zoller, Phys. Rev. Lett. 95, 105701 (2005); B. Damski, Phys. Rev. Lett. 95, 035701 (2005).

[5] A. Polkovnikov, Phys. Rev. B 72, 161201(R) (2005).

[6] J. Dziarmaga, Phys. Rev. Lett. 95, 245701 (2005).

[7] B. Damski and W. H. Zurek, Phys. Rev. A 73, 063405 (2006).

[8] R. W. Cherng and L. S. Levitov, Phys. Rev. A 73, 043614 (2006).

[9] J. Dziarmaga, Phys. Rev. B 74, 064416 (2006); T. Caneva, R. Fazio, and G. E. Santoro, Phys. Rev. B 76, 144427 (2007).

[10] V. Mukherjee, U. Divakaran, A. Dutta, and D. Sen, Phys. Rev. B 76, 174303 (2007); U. Divakaran, A. Dutta, and D. Sen, Phys. Rev. B 78, 144301 (2008).

[11] U. Divakaran, V. Mukherjee, A. Dutta, and D. Sen, J. Stat. Mech. Theory and Experiment P02007 (2009); S. Deng, G. Ortiz and L. Viola, Euro. Phys. Lett 84, 67008 (2008).

[12] A. Polkovnikov and V. Gritsev, Nature Physics 4, 477 (2008)
[13] K. Sengupta, D. Sen, and S. Mondal, Phys. Rev. Lett. 100, 077204 (2008); S. Mondal, D. Sen, and K. Sengupta, Phys. Rev. B 78, 045101 (2008).

[14] D. Patane, A. Silva, L. Amico, R. Fazio, and G. Santoro, Phys. Rev. Lett,101, 175701 (2008).

[15] P. Zanardi and N. Paunkovic, Phys. Rev. E 74, 031123 (2006); Ho-Man Kwok, Wen-Qiang Ning, Shi-Jian Gu and Hai-Qing Lin, Phys. Rev. E 78, 032103 (2008).

[16] T. W. B. Kibble, J. Phys. A 9, 1387 (1976), Phys. Rep. 67, 183 (1980).

[17] W. H. Zurek, Nature (London) 317, 505 (1985), and Phys. Rep. 276, 177 (1996).

[18] I. Bloch, J. Dalibard, and W. Zwerger, Rev. Mod. Phys. 80, 885 (2008).

[19] E. Lieb, T. Schultz, and D. Mattis, Ann. Phys. (NY) 16, 407 (1961); J. E. Bunder and R. H. McKenzie, Phys. Rev. B 60, 344 (1999).

[20] A. Kitaev, Ann. Phys. (N.Y.), 3212 (2006).

[21] C. Zener, Proc. Roy. Soc. London Ser A 137, 696 (1932); L. D. Landau and E. M. Lifshitz, Quantum Mechanics: Non-relativistic Theory, 2nd ed. (Pergamon Press, Oxford, 1965).

[22] S. Suzuki and M. Okada, in Quantum Annealing and Related Optimization Methods, Ed. by A. Das and B. K. Chakrabarti (Springer-Verlag, Berlin, 2005), p. 185.

[23] I. Titvinidze and G. I. Japaridze, Eur. Phys. J B 32383 (2003); A. A. Zvyagin and G. A. Skorobagat'ko, Phys. Rev. B 73, 024427 (2006).

[24] M. Fabrizio, Phys. Rev. B 54, 10054 (1996); R. Arita, K. Kuroki, H.Aoki and M. Fabrizio, Phys. Rev. B 57, 10324 (1996). 